\title{
Calculation and Experimental Validation of Pressure and Temperature Effects on COG-Air Fuel Mixtures
}

\author{
Skrinsky Jan
}

Energy Research Centre, VŠB-TU Ostrava, 17. Listopadu 15/2172, 70833 Ostrava, Czech Republic

\begin{abstract}
COG have been widely used together with blast furnace gas and blast furnace oxygen gas in the steel industry in Moravian-Silesian region of Czech Republic. COG is a flammable and explosive substance. Most explosion characteristics published so far are valid for pure compounds and limited experimental conditions, mostly ambient. There have been no explosion characteristic exists for COG-air mixtures which cover industrial conditions up to $423 \mathrm{~K}$. Experimental tests have been carried out in a $20-\mathrm{L}$ closed explosion chamber adopted for the explosion tests. The element potential approach in the thermochemical equilibrium calculations applied in the Chemkin subroutine has been used for explosion pressure calculations. Different explosion characteristics have been reported in a range from $298 \mathrm{~K}$ up to $423 \mathrm{~K}$ and from 0.5 bar(a) up to 1.0 bar(a).
\end{abstract}

\section{Introduction}

All process industry accidents fall under three broad categories fire, explosion, and toxic release. Of these, fire is the most common, followed by explosions. Within these categories explosion make the immediate damage potential [1].

Coke oven gas $(\mathrm{COG})$ is highly rated as a valuable by-product of coal carbonization to produce coke in the steel industry [2].

Although COG is regarded as a non-standard gaseous fuel, it still has a reasonable energy content and calorific value, which depend on the nature of coal and the type of carbonization and have been widely used together with blast furnace gas and converter gas in the steel industry in Moravian-Silesian region of Czech Republic. However, coke oven gas is a flammable and explosive substance. Improper operation in the process of production, usage, transportation and storage can easily lead to combustion and explosion, posing a serious threat to lives and property. Therefore, how to safely and effectively utilize or dispose of coke oven gas has become an urgent task [3].

There are no explosion characteristic exists for COG which cover all conditions occurring in "real life" initial conditions. Most explosion characteristics published so far are valid for specific and limited conditions. Few data at standard conditions are available in open literature [4]. In the presented paper these characteristics have been systematically examined and determined in the $20-\mathrm{L}$ explosion chamber for the first time.

\section{Experiment}

\subsection{Experimental device}

Measurements of the $\mathrm{P}_{\max }$ and $(\mathrm{dP} / \mathrm{dT})_{\max }$ have been performed in the 20-L explosion chamber (OZM Research s.r.o., Czech Republic). The spherical explosion chamber allows the measurement of parameters of explosive gas dispersion in accordance with the terms and specifications in EN 15967 [5]. The mixing process proceeds inside the explosion chamber. The gasses are dosed into the chamber from the dosing vessel equipped by three inputs for connection to cylinders with nitrogen and oxygen and line for free suction of air from outside. The ignition source is located in the geometrical centre of the cylinder. In the experiment, ignition was performed with hot wire between stainless steel electrodes.

\subsection{Experimental procedure}

The experimental procedure was as follows: (a) the air in the explosion vessel was evacuated to a vacuum of 0.1 mbar; (b) mixing gases were added into the vessel at different ratios; (c) the mixture was admitted at the desired pressure, then ignited at once; (d) after ignition and the capture of the signals by the acquisition system, the burned gas was completely evacuated. Then, a new cycle was repeated.

\footnotetext{
Corresponding author: author@e-e-mail.org
} 


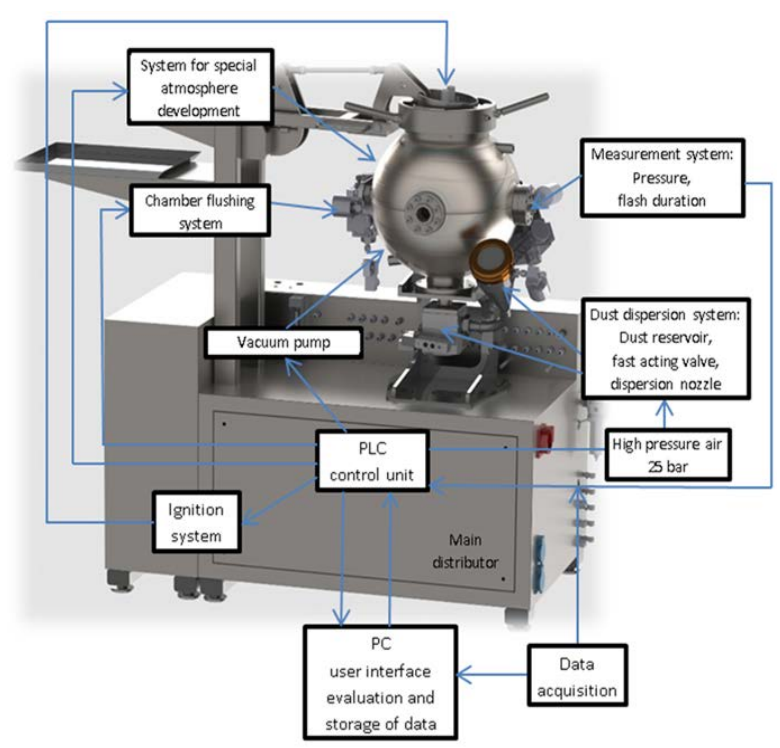

Fig. 1. Spherical vessel experimental arrangement.

\section{Calculation}

Calculation procedure is described in [3]. The element potential approach in the thermochemical equilibrium calculations applied in the Chemkin subroutine has been used for explosion pressure calculations. This approach represents 'ideal' deflagrations in closed systems well and gives the highest possible attainable explosion pressures. Explosion pressures are calculated using the species and their thermodynamic values from the GRI 3.0.

The maximum pressure rise rate during gas explosions in enclosures, $(\mathrm{dP} / \mathrm{dt})_{\max }$, and the deflagration index, $\mathrm{K}_{\mathrm{G}}$, are important explosion characteristics of premixture. They are used to quantify the potential severity of an explosion.

The maximum pressure rise rate, $(\mathrm{dP} / \mathrm{dt})_{\max }$, depends not only on the mixture properties (such as mixture composition, initial temperature and initial pressure) but also on the volume of the vessel in which gas explosion takes place. Unlike $(\mathrm{dP} / \mathrm{dt}) \max$, the deflagration index is an intrinsic property of the premixture and it is independent of the volume of the vessel used in experimental measurements.

The relationship between $\mathrm{K}_{\mathrm{G}}$ and $(\mathrm{dP} / \mathrm{dt})_{\max }$ is given by $\operatorname{Eq}(1)$ :

$$
K_{G}=(d P / d t)_{\max } V^{l / 3}
$$

in which $\mathrm{K}_{\mathrm{G}}$ is the deflagration index (bar.m/s), $\mathrm{V}$ is the vessel volume $\left(\mathrm{m}^{3}\right), \mathrm{dP} / \mathrm{dT}$ is the maximum rate of pressure rise $(\mathrm{bar} / \mathrm{s})$. In order to capture the intrinsic reactivity of COG mixture, the burning velocity has been obtained from time pressure records of explosions occurring in 20-L closed explosion chamber.

The burning velocity in $\mathrm{Eq}(3)$ first published by [6] has been calculated from the pressure time history by using the time derivative of flame radius, $r_{f}$, as given by the correlation in $\mathrm{Eq}(2)$ : $r_{f}=(3 V / 4 \pi)^{1 / 3}\left[1-\left(P_{0} / P\right)^{1 / \gamma}\left(\left(P_{\max }-P\right) /\left(P_{\max }-P_{0}\right)\right)\right]^{1 / 3}$

in which $r_{f}$ is the flame radius $(m), V$ is the vessel volume $\left(\mathrm{m}^{3}\right), \mathrm{P}_{0}$ is the initial pressure (bar), $\mathrm{P}$ is the actual pressure (bar), $\mathrm{P}_{\max }$ is the maximum explosion pressure (bar) and $\gamma$ the adiabatic coefficient of the unburned gas (-).

$$
\begin{gathered}
s=\llbracket 1 /\left(\left(P_{\max }-P_{0}\right)\right)^{1 / 3}(4 \pi / 3 V)^{-1 / 3}\left(P / P_{0}\right)^{1 / \gamma} \\
{\left[1-\left(P / P_{0}\right)^{1 / \gamma}\left(\left(P_{\max }-P\right) /\left(P_{\max }-P_{0}\right)\right)\right]^{-2 / 3} d P / d t}
\end{gathered}
$$

in which $\mathrm{s}$ is burning velocity $(\mathrm{m} / \mathrm{s}), \mathrm{r}_{\mathrm{f}}$ is the flame radius $(\mathrm{m}), \mathrm{R}$ is specific gas constant $(\mathrm{J} / \mathrm{kg} . \mathrm{K}), \mathrm{V}$ is the vessel volume $\left(\mathrm{m}_{3}\right), \mathrm{P}_{0}$ is the initial pressure (bar), $\mathrm{P}$ is the actual pressure (bar), $\mathrm{P}_{\max }$ is the maximum explosion pressure (bar), $\gamma$ the adiabatic coefficient of the unburned gas $(-)$ and $\mathrm{dP} / \mathrm{dT}$ is the rate of pressure rise (bar/s).

\section{Results and discussion}

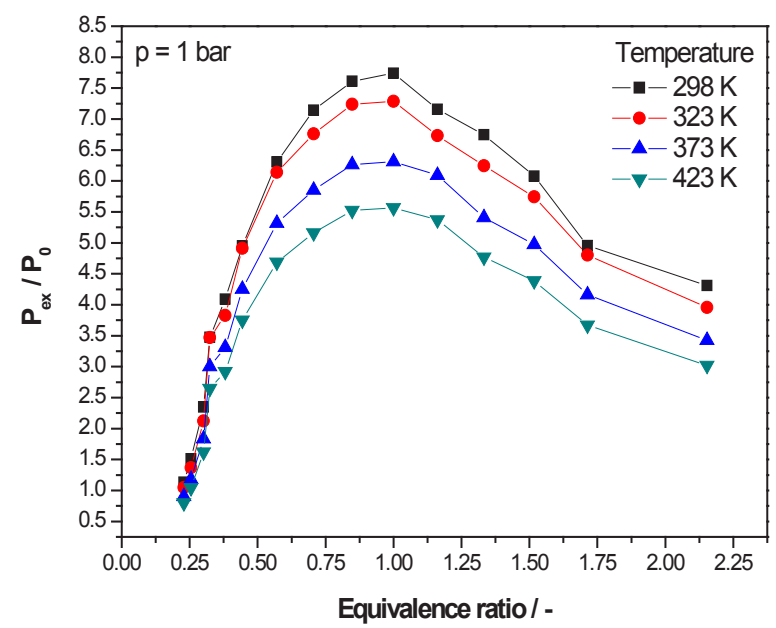

Fig. 2. Normalized peak explosion pressure $\left(\mathrm{P}_{\max } / \mathrm{P}_{0}\right)$ versus equivalence ratio at atmospheric pressure and four initial temperatures.

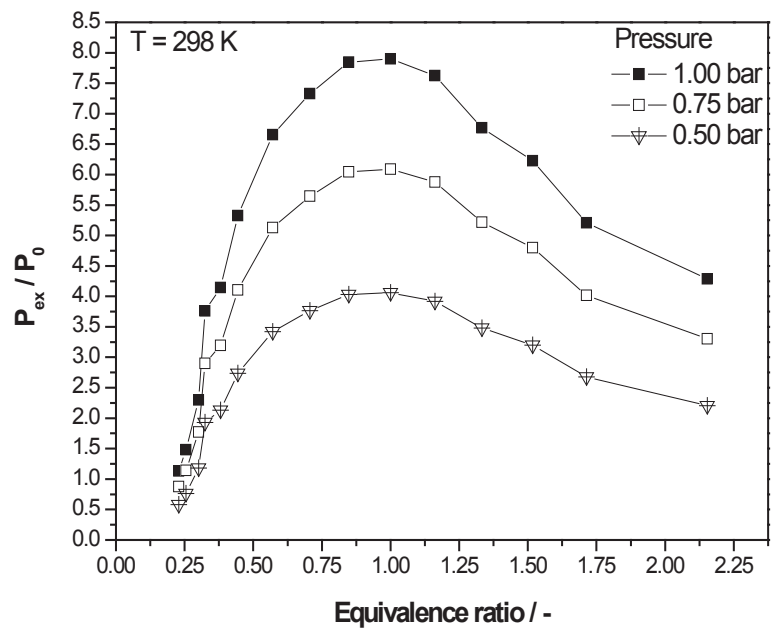

Fig. 3. Normalized peak explosion pressure $\left(\mathrm{P}_{\max } / \mathrm{P}_{0}\right)$ versus equivalence ratio at atmospheric temperature and three initial pressures.

As observed from Fig. 2-3 the experimental results, explosion pressure shows a certain regularity with the variation of coke oven gas concentration, and the 
maximum pressure achieves its peak value near the stoichiometric concentration (The calculated value based on the compositions of coke oven gas is $20.9 \%$.) and tends to decrease if the concentration is lower or higher than $20.9 \%$. This pattern forms because in the vicinity of the stoichiometric concentration, the coke oven gas and oxygen can be fully utilised, causing the most intense reaction to occur, and thus, the largest pressure value is generated. When the concentration of coke oven gas is less than the stoichiometric concentration, even though there is surplus oxygen, the coke oven gas is also in relatively shorter supply, so the explosion is relatively weaker, correspondingly producing relatively less pressure. When concentration of coke oven gas is more than the stoichiometric concentration, the oxygen concentration will relatively low. Thus, the coke oven gas concentration actually involved in the reaction is lower, so less pressure is produced. The greater the concentration of coke oven gas, the less oxygen content, and therefore the less of the coke oven gas actually participates in the reaction. Thus, the pressure becomes smaller.

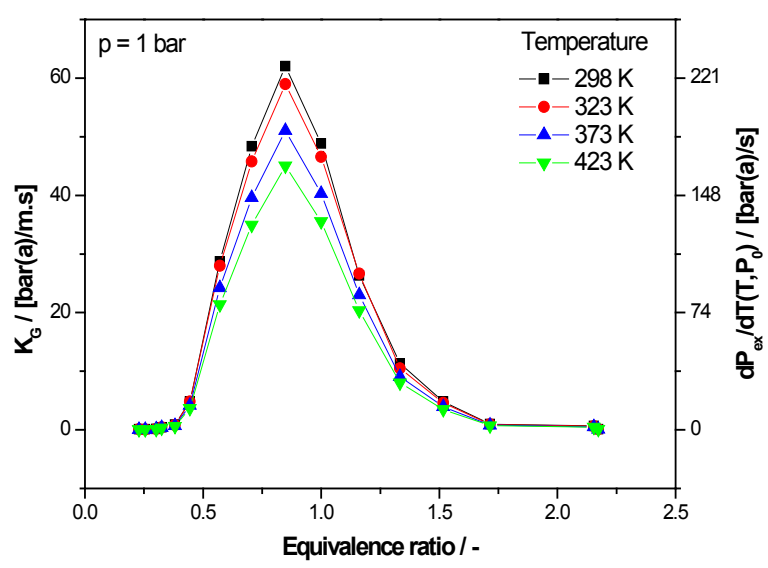

Fig. 4. Rate of pressure rise, $(\mathrm{dP} / \mathrm{dt})_{\mathrm{ex}}$, and deflagration index, $\mathrm{K}_{\mathrm{G}}$, versus equivalence ratio at elevated temperatures and pressures.

Fig. 4 shows the comparison among rate of pressure rise, $(\mathrm{dP} / \mathrm{dt})_{\mathrm{ex}}$, and deflagration index, $\mathrm{K}_{\mathrm{G}}$, versus equivalence ratio at elevated temperatures and pressures for the methanol/air mixtures. Maximum rate of pressure rise and deflagration index reach their peaks at $\Phi$ around 1.46 , and they decrease at both lean and rich mixtures. As shown in Fig. 4, $(\mathrm{dP} / \mathrm{dt})_{\max }$ and $\mathrm{K}_{\mathrm{G}}$ give an approximate value at varied initial temperatures, indicating that $(\mathrm{dP} / \mathrm{dt})_{\max }$ and $\mathrm{K}_{\mathrm{G}}$ are sensitive to the variation of temperature $(109,102,98,90 \mathrm{bara} / \mathrm{m} . \mathrm{s})$. Rate of pressure rise is affected by both flame speed and heat release. Flame speed increases monotonically with the increase of temperature, but the decrease of total fuel mass results in the decreased heat release and offsets the effect of flame speed on the explosion pressure. With the increase of initial pressure $(\mathrm{dP} / \mathrm{dt})_{\max }$ and $\mathrm{K}_{\mathrm{G}}$ increase dramatically, especially around equivalence ratio of 1.1. This differs from the flame speed, but agrees with the mass burning flux when taking into account of the effect of density. It is noted that deflagration index is less than
$20 \mathrm{MPams}^{-1}$ at $\Phi=1.0$ and the initial pressure of 0.1 $\mathrm{MPa}$, belonging to the first class of deflagration index and low potential of explosion hazard! However, KG could exceeds $30 \mathrm{MPa} \mathrm{m} \mathrm{s}-1$ around the stoichiometric ratio at the pressures higher than $0.1 \mathrm{MPa}$ and could enter the highest class of deflagration index.

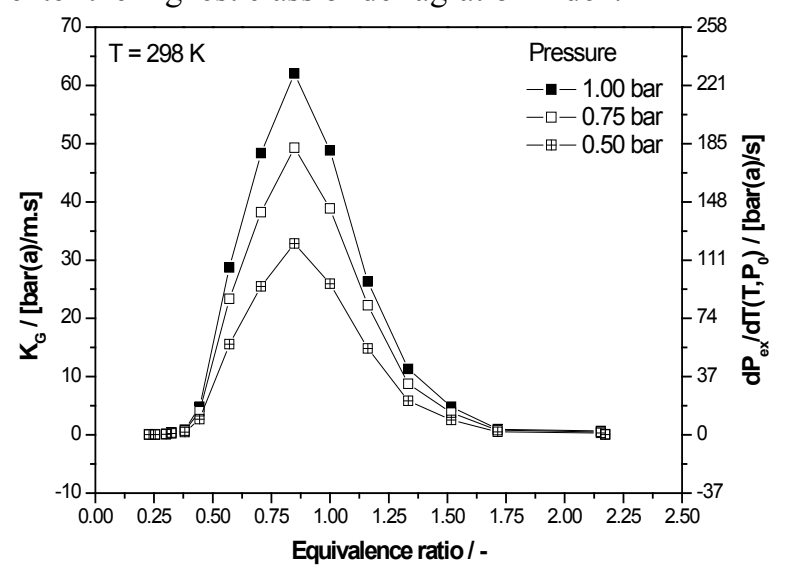

Fig. 5. Rate of pressure rise, $(\mathrm{dP} / \mathrm{dt})_{\mathrm{ex}}$, and deflagration index, $\mathrm{K}_{\mathrm{G}}$, versus equivalence ratio at elevated temperatures and pressures.

The maximum rate of pressure rise and the maximum explosion pressure have similar relationships with coke oven gas concentration (as plotted in Fig. 5.). When the concentration of coke oven gas is lower than its stoichiometric concentration, oxygen is relatively abundant. With the increase of coke oven gas concentration, its reaction volume per unit time increases, so the maximum rate of pressure rise also increases, and it achieves the maximum value near the stoichiometric concentration $(\Phi=0.9)$. However, when the coke oven gas concentration is higher than the stoichiometric concentration, increasing the coke oven gas concentration requires the oxygen content in the mixture to decline, so the amount of coke oven gas actually involved in the reaction is reduced correspondingly.

\section{Conclusion}

An experimental study on the characteristics of COG-air explosions in a $20-\mathrm{L}$ closed spherical vessel with central ignition was carried out, using COG-air mixtures with variable composition, at various initial temperatures and pressures. The main findings are summarized as follows:

1. Different explosion characteristics have been reported in a range from $298 \mathrm{~K}$ up to $423 \mathrm{~K}$ and from 0.5 bar(a) up to 1.0 bar(a)

2. The explosion pressures of COG-air mixtures attained their highest value at a concentration 1.0 within the studied concentration range of 0.25-2.25.

3. Most reliable ("worse case") explosion pressure is the pmax: 8.2 bar(a) for $298 \mathrm{~K}, 7.7$ bar(a) for $323 \mathrm{~K}, 6.3$ bar(a) for $353 \mathrm{~K}$ and 5.6 bar(a) for $423 \mathrm{~K}$.

4. The explosion pressure, the maximum rate of pressure rise, the deflagration index are decreased, while the 
normalized mass burning rate is increased with the increase of initial temperature.

5. The increase of initial temperature, the peak explosion pressure, combustion duration and flame development period all linearly decrease, while the maximum rate of pressure rise varies little.

This work would not have been possible without the financial support of Innovation for Efficiency and Environment Growth, reg. no. LO1403 supported by National Programme for Sustainability and financed by the Ministry of Education, Youth and Sports.

\section{References}

1. M. Skřínská, J. Skřínský, P. Dolníček, P. Lukešová, , R. Přichystalová, Ch. Serafínov Mat. Sci. For., 811 (2015).

2. R. Razzaq, Ch. Li, S. Zhang China Fuel 113 (2013).

3. J. Skrinsky, J. Veres, V. Peer, P. Friedel, J. Travnickova, A. Dalecka Chem. Eng. Trans. 52 (2016)

4. Z. Zhang, B. Lin, G. Li, Q. Ye Safety Science 55 (2013)

5. EN 15967, 2012. Determination of maximum explosion pressure and the maximum rate of pressure rise of gases and vapours. European Standard, Beuth Verlag, Berlin Wien Zurich.

6. Dahoe A. E., Zevenbergen J. F., Verheijen P. J. T., Lemkowitz S.M., Scarlett B. J. Loss Prev. Process 9 (1996) 